\title{
SCHUR CONVEXITY OF STOLARSKY'S EXTENDED MEAN VALUES
}

\section{K. Murali and K. M. Nagaraja}

Abstract. In the recent years, the Schur convexity and Schur geometrically convexity of Stolarsky's mean values have grabed the focus of many mathematicians and researchers. In this article, the Schur convexity of Stolarsky's extended type mean values are discussed.

Mathematics subject classification (2010): Primary 26D10, 26D15.

Keywords and phrases: Heron mean, harmonic mean, Schur concavity, convexity.

\section{REFERENCES}

[1] P. S. Bullen, Handbook of means and their inequalities, Kluwer Acad. Publ., Dordrecht, 2003.

[2] Y. M. Chu, X. M. ZHANG AND G. D. WANG, The Schur geometrical convexity of the extended mean values, J. Convex. Anal., 15 (2008), No. 4, 707-718.

[3] Y. M. ChU AND X. M. ZHANG, Necessary and sufficient conditions such that extended mean values are Schur-convex or Schur-concave, J. Math. Kyoto Univ., 48 (2008), No. 1, 229-238.

[4] Y. M. CHU AND Y. P. LV, The Schur harmonic convexity of the Hamy symmetric function and its applications, J. Inequal. Appl., Vol. 2009, Art. ID 838529, 10 pages, doi:10.1155/2009/838529; available online at http://downloads.hindawi.com/journals/jia/2009/838529.pdf.

[5] G. H. Hardy, J. E. Littlewood ANd G. Pólya, Some simple inequalities satisfied by convex functions, Messenger Math., 58 (1929), 145-152.

[6] E. B. Leach And M. C. Sholander, Extended mean values II, J. Math. Anal. Appl., 92 (1983), 207-223.

[7] D.-M. LI AND H. N. SHI, Schur convexity and Schur-geometrically concavity of generalized exponent mean, J. Math. Inequal., 3 (2009), no. 2, 217-225.

[8] V. Lokesha, S. Padmanabhan, K. M. Nagaraja and Yilmaz Simsek, Relation between Greek means and various other means, General Mathematics, 17 (3) (2009), 3-13.

[9] V. Lokesha, K. M. Nagaraja, Naveen Kumar B and S. Padmanabhan, Oscillatory type mean in Greek means, Int. e-Journal of Engg. Maths Theory and Applications, 9 (3) (2010), 18-26.

[10] A. W. Marshall AND I. OlKIN, Inequalities: Theorey of Majorization and Its Applications, New York, Academic Press, 1979.

[11] K. M. Nagaraja, K. Murali And V. Lokesha, Schur convexity and concavity of Gnan mean, Proceedings of the Jangjeon Math. Society. 17 (2014), no. 3, pp. 355-367.

[12] K. M. Nagaraja And Sudhir Kumar SAHU, Schur Geometric convexity of Gnan mean for two variables, Journal of the International Mathematical Virtual Institute, Vol. 3 (2013), 39-59.

[13] K. M. Nagaraja, P. Siva Kota Reddy and K. Sridevi, Schur Harmonic convexity of Gnan mean for two variables, Journal of the International Mathematical Virtual Institute, Vol. 3 (2013), $61-80$.

[14] K. M. Nagaraja, P. Siva Kota Reddy and B. Naveenkumar, Refinement of Inequality involving ratio of Means for four Positive Arguments, Bulletin of International Mathematical Virtual Institute, Vol. 3 (2013), 135-138.

[15] K. M. Nagaraja, V. Lokesha and S. Padmanabhan, A simple proof on strengthening and extension of inequalities, Advn. Stud. Contemp. Math., 17 (1) (2008), 97-103.

[16] K. M. Nagaraja And Sudhir Kumar Sahu, Schur harmonic convexity of Stolarsky extended mean values, Scientia Magna.2013.

[17] F. QI, J. SÁNDOR AND S. S. DRAGOMIR, Notes on the Schur-convexity of the extended mean values, Taiwanese J. Math., 9 (2005), no. 3, 411-420. 
[18] F. QI, A note on Schur-convexity of extended mean values, Rocky Mountain J. Math., 35 (2005), no. $5,1787-1793$.

[19] H. N. SHI, S. H. WU AND F. QI, An alternative note on the Schur-convexity of the extended mean values, Math. Inequal. Appl., 9 (2006), no. 2, 219-224.

[20] Slavko Simic, An extension of Stolarsky means, Novi Sad J. Math. Vol. 38, no. 3, 2008, 81-89.

[21] Slavko Simic, On weighted Stolarsky means, Sarajevo Journal of Mathematics, Vol. 7, no. 19, 2011, 3-9.

[22] K. B. Stolarsky, Generalizations of the Logarithmic Mean, Math. Mag., 48 (1975), 87-92.

[23] K. B. Stolarsky, The power and generalized Logarithmic Means, Amer. Math. Monthly, 87 (1980), $545-548$.

[24] X. M. Zhang, Geometrically Convex Functions, Hefei, An. hui University Press, 2004. (Chinese).

[25] Zhen-Hang Yang, Necessary and Suffcient Condition for Schur Convexity of the Two-Parameter Symmetric Homogeneous Means, Applied Mathematical Sciences, Vol. 5, 2011, no. 64, 3183-3190. 